\title{
LETTER
}

Immunotherapy

\section{Single agent talacotuzumab demonstrates limited efficacy but considerable toxicity in elderly high-risk MDS or AML patients failing hypomethylating agents}

\author{
Anne Sophie Kubasch ${ }^{1,2,3} \cdot$ Freya Schulze ${ }^{2,3,4} \cdot$ Aristoteles Giagounidis $^{2,3,5} \cdot$ Katharina S. Götze $^{2,3,6}$. \\ Jan Krönke $\mathbb{D}^{2,3,7} \cdot$ Katja Sockel $^{2,3,4} \cdot$ Jan Moritz Middeke ${ }^{2,3,4} \cdot$ Fatiha Chermat $^{3,8} \cdot$ Silke Gloaguen $^{2,3}$. \\ Martin Puttrich ${ }^{3,9}$ - Carmen Weigt ${ }^{3,9} \cdot$ Doreen William $^{10,11}$ - Pierre Fenaux ${ }^{3,8,12}$ - Richard F. Schlenk ${ }^{3,11,13}$. \\ Christian Thiede $^{3,4} \cdot$ Sebastian Stasik ${ }^{3,4} \cdot$ Anna Mies $^{3,4} \cdot$ Lionel Adès $^{3,8,12} \cdot$ Uta Oelschlägel $^{2,3,4} \cdot$ Uwe Platzbecker $^{1,2,3}$
}

Received: 22 July 2019 / Revised: 17 October 2019 / Accepted: 3 November 2019 / Published online: 3 December 2019

(c) Springer Nature Limited 2019

\section{To the Editor:}

First line standard therapeutic approaches in elderly highrisk (HR) MDS or AML patients are largely based on hypomethylating agents (HMAs) including azacitidine (AZA) or decitabine (DAC), but responses are generally short-lived and occur only in $50-60 \%$ of patients [1, 2]. Patients failing HMAs have a dismal prognosis with a median survival of around 5 months [3]. The high unmet medical need for new treatment options makes this patient population an important target of ongoing clinical research.

CD123 (Interleukin-3 receptor alpha chain [IL3RA]) is a potential target for antibody- or cell-based therapies directed towards leukemic stem cells (LSCs) [4], because CD123 is overexpressed on leukemic blasts and an important growth

These authors contributed equally: Anne Sophie Kubasch, Freya Schulze and differentiation receptor for early LSCs [5]. Upregulated expression of CD123 has been associated with higher blast cell counts at diagnosis and poorer complete response (CR) and survival rates in AML [6]. Moreover, the CD123 expression is low/absent on normal hematopoietic stem cells, making CD123 an attractive and specific target for immunotherapy-based approaches [7,8]. Talacotuzumab (TAL, JNJ-56022473) is an IgG1 monoclonal antibody targeting CD123 preferentially via antibody-dependent cellular cytotoxicity (ADCC) mediated by natural killer (NK) cells [9] and has been shown to induce potent in vitro ADCC against IL3RA-expressing AML blasts/LSC and to reduce leukemic cell growth in murine xenograft models of human AML [10]. In addition, the antibody inhibits signaling by IL-3, the main ligand of CD123, to reduce the proliferation of leukemic progenitor cells [11].

The SAMBA trial, a phase II investigator-initiated study (NCT02992860) of the German and French MDS study groups within the EMSCO network, assessed as primary endpoint the overall hematological response rate (CR,

7 Department of Internal Medicine III, University Hospital Ulm, Ulm, Germany

8 Groupe Francophone des Myélodysplasies, Paris, France

9 GWT-TUD GmbH, Dresden, Germany

10 Institute for Clinical Genetics, Faculty of Medicine Carl Gustav Carus, TU Dresden, Dresden, Germany

11 National Center for Tumor Diseases (NCT), Heidelberg, Germany

12 Service d'Hématologie Seniors, Assistance Publique-Hôpitaux de Paris and Paris University, Paris, France

13 Department of Internal Medicine V, Heidelberg University Hospital, Heidelberg, Germany 
partial response (PR), marrow $\mathrm{CR}$ (CRi), or hematological improvement (HI)) according to IWG 2006 criteria [12] after 3 months of single agent TAL treatment in elderly HRMDS (IPSS intermediate II and high risk) and AML patients failing HMA therapy. Dose and dosing schedule based on PK, PD, and safety data was generated in the prior phase I study (NCT01632852). TAL was given IV at a dose of $9 \mathrm{mg} / \mathrm{kg}$ once every 2 weeks $(14 \mathrm{~d} \pm 2 \mathrm{~d}$ ) for a total of 6 infusions over 3 months. Responders could go on to receive up to 20 additional infusions, whereas for nonresponders initial treatment with TAL was followed by an up to 9 months observation period without further treatment.

The study was initially powered to include a total of 43 patients, but the SAMBA trial was prematurely terminated because the manufacturer decided to stop the entire TAL study program due to lack of efficacy and high toxicity rates in a pivotal study involving TAL in combination with DAC as first line treatment in AML (NCT02472145) [13]. Consequently, only 24 patients (19 AML and 5 HR-MDS) with a median age of 77 years (range 71-90) who either failed to achieve CR, PR, HI, or relapsed after at least six AZA or four DAC treatment cycles were included in the study from November 2016 until June 2017. Informed consent was obtained from all included patients prior start of TAL treatment. Among the 24 included patients, 16 patients $(66.6 \%)$ were resistant to prior HMA therapy and 8 patients (33.3\%) relapsed after initially HMA response. Patient disposition is provided in Table 1 , including molecular genetics at study entry in line with a poor-risk and advanced study population.

We observed significant toxicities after TAL treatment, most frequent severe adverse events were infections $(n=26)$, cytopenias $(n=6)$, cardiac and gastrointestinal disorders $(n=$ 6 , each), infusion-related reactions $(n=4)$ as well as nervous system disorders $(n=3)$ including one patient with cerebral infarction and one patient with reversible Guillain-Barré syndrome. Due to high treatment-related toxicity only 10 patients $(42 \%)$ reached the planned response assessment after 3 months, with the remaining 14 patients (58\%) being assessed for response after 2 months of treatment. Reasons for premature study discontinuation in these 14 patients were disease progression $(n=4)$, adverse event $(n=1)$, withdrawal of informed consent $(n=2)$, investigator decision $(n=1)$, and death $(n=6)$, two of these patients died from pneumonia possibly related to treatment toxicity.

The 4 weeks mortality rate was $20.8 \%(n=5)$ and the 8 weeks mortality rate $25 \%(n=6)$. The overall response rate was $8.3 \%(n=2)$ including one complete remission with incomplete hematologic recovery (CRi) and one erythroid hematologic improvement (HI-E). Moreover, three additional patients experienced disease stabilization (SD) after TAL treatment according to IWG 2006 response criteria [12]. Response duration was 22 weeks (CRi) and
Table 1 Patient baseline characteristics in the SAMBA trial

\begin{tabular}{|c|c|}
\hline Patient characteristics, $n,(\%)$ & $n=24$ \\
\hline Age (years), median (IQR) & $77(71-90)$ \\
\hline AML & $19(79 \%)$ \\
\hline MDS & $5(21 \%)$ \\
\hline \multicolumn{2}{|l|}{ Cytogenetics, $n,(\%)$} \\
\hline Normal karyotype & $12(50 \%)$ \\
\hline Abnormal karyotype & $12(50 \%)$ \\
\hline Complex karyotype ( $\geq 3)$ & $5(21 \%)$ \\
\hline \multicolumn{2}{|c|}{ ELN 2017 AML risk classification $(n=19)$} \\
\hline Favorable & $2(10 \%)$ \\
\hline Intermediate & $8(43 \%)$ \\
\hline Unfavorable & $9(47 \%)$ \\
\hline \multicolumn{2}{|l|}{ Somatic mutations, $n,(\%)$} \\
\hline No mutation & $2(8 \%)$ \\
\hline$\geq 1$ mutation & $22(92 \%)$ \\
\hline$\geq 2$ mutations & $16(67 \%)$ \\
\hline$\geq 3$ mutations & $15(63 \%)$ \\
\hline \multicolumn{2}{|l|}{ Epigenetic } \\
\hline ASXL1 & $7(29 \%)$ \\
\hline TET2 & $6(25 \%)$ \\
\hline $\mathrm{EZH} 2$ & $4(17 \%)$ \\
\hline DNMT3A & $3(13 \%)$ \\
\hline IDH1 & $3(13 \%)$ \\
\hline BCORL1 & $2(8 \%)$ \\
\hline IDH2 & $2(8 \%)$ \\
\hline BCOR & $2(8 \%)$ \\
\hline \multicolumn{2}{|l|}{ Cohesin } \\
\hline STAG2 & $4(17 \%)$ \\
\hline RAD21 & $1(4 \%)$ \\
\hline \multicolumn{2}{|l|}{ Splicing } \\
\hline SRSF2 & $4(17 \%)$ \\
\hline SF3B1 & $2(8 \%)$ \\
\hline U2AF1 & $2(8 \%)$ \\
\hline ZRSR2 & $2(8 \%)$ \\
\hline \multicolumn{2}{|l|}{ Transcription factors } \\
\hline RUNX1 & $3(13 \%)$ \\
\hline CEPBA & $3(13 \%)$ \\
\hline CUX1 & $1(4 \%)$ \\
\hline GATA2 & $1(4 \%)$ \\
\hline \multicolumn{2}{|l|}{ TP53 } \\
\hline TP53 & $7(29 \%)$ \\
\hline \multicolumn{2}{|l|}{ Signaling } \\
\hline NRAS & $2(8 \%)$ \\
\hline KRAS & $2(8 \%)$ \\
\hline PTPN11 & $2(8 \%)$ \\
\hline JAK2 & $1(4 \%)$ \\
\hline \multicolumn{2}{|l|}{ Others } \\
\hline NPM1 & $2(8 \%)$ \\
\hline PHF6 & $2(8 \%)$ \\
\hline KDM6A & $1(4 \%)$ \\
\hline NOTCH1 & $1(4 \%)$ \\
\hline \multicolumn{2}{|l|}{ Blood counts, median, (range) } \\
\hline WBC (/nl) & $2.51(0.37-12.64)$ \\
\hline Hemoglobin $(\mathrm{g} / \mathrm{dl})$ & $9.25(8.05-11.7)$ \\
\hline PLT (/nl) & $28(4-1018)$ \\
\hline ANC $(/ \mathrm{nl})$ & $0.59(0.0-5.59)$ \\
\hline Lymphocytes (/nl) & $0.80(0.024-4)$ \\
\hline
\end{tabular}




\begin{tabular}{lc} 
Table 1 (continued) & \\
\hline Patient characteristics, $n,(\%)$ & $n=24$ \\
\hline BM blasts $(\%)$ & $27(7-89)$ \\
Interval from diagnosis (years) & $1.76(0.61-7.82)$ \\
Therapy outcome, median, (range) & \\
Days on treatment & $57(1-374)$ \\
Cycles completed & $3(1-26)$ \\
\hline
\end{tabular}

8 weeks (HI-E), respectively. The median duration of treatment was 57 days (range 1-374 days) and median overall survival for the entire patient cohort was 3.6 months (range $<1.0-16.5$ months). Six patients showed a reduction in blast percentage in bone marrow (BM) during the study, while others displayed no change or an increase in BM blasts during treatment (Fig. 1a).

The one patient achieving CRi after three doses of TAL treatment was an AML patient with initially 24\% BM blasts and an adverse risk cytogenetic profile harboring a complex karyotype $(-5 ;-7 ;-17 /$ abnl $(17 p))$, molecular analysis using NGS revealed an isolated TP53 mutation. The patient achieving HI-E after four doses of TAL treatment was an AML patient with $30 \%$ BM blasts at screening and normal karyotype but mutated NPM1, DNMT3A, and RAD21.

The study was accompanied by immune monitoring via flow cytometry to investigate the distribution of T-, NK cells, blasts (CD34+ and CD117+), monocytes and myeloid-derived suppressor cells (MDSCs) in peripheral blood $(\mathrm{PB})$ and $\mathrm{BM}$ at the time of screening and during therapy. Before treatment initiation, patients $(n=24)$ displayed a disturbed immune profile compared with agedmatched healthy controls (HC, $n=24)$. The activation state of NK cells is determined by the balance of stimulatory and inhibitory receptors [14]. Compared with HC, patients expressed lower levels of CD56dim NK cells in PB (73 vs. $84 \%$ of NK cells; $p=0.065$ ) (Fig. 1b), significantly higher levels of inhibiting NK-cell receptors such as KIR2DL2 ( 8.8 vs. $3.5 \%$ of NK cells; $p=0.005$ ) and lower levels of activating NK-cell receptors such as NKG2D (93 vs. 99\% of NK cells; $p=0.004)$. These findings are in line with previous studies demonstrating an association between impaired NK-cell activity in MDS or AML patients and higher International Prognostic Score (IPSS), abnormal karyotype, excess of BM blasts and BM hypercellularity [15]. Patients also showed an increased number of $\mathrm{T}$ regulatory cells (Tregs) in PB compared with HC (Tregs 2.32 vs. $1.59 \%$ of leukocytes; $p=0.01$ ) whereas patients had a significantly less median total leukocyte count compared to HC $(2.41 / \mathrm{nl}$ vs. $6.36 / \mathrm{nl}, p=0.002)$. Interestingly, those patients with a reduction in BM blast count after TAL treatment (Fig. 1a) exhibited initially a significant higher percentage of Tregs in PB compared to nonresponders (4.79 vs. $2.46 \%$ of leukocytes; $p=0.049)$. Overall, absolute concentration of Tregs in PB (1-70 cells/ $\mu \mathrm{l})$ and circulating blasts counts were highly variable in patients $(0.01-76.69 \%$ of leukocytes, mean $10.37 \%$ ).

Moreover, compared to nonresponders, responders showed a significantly higher percentage of absolute CD8 $+\mathrm{T}$-cell count in PB (46.41 vs. $23.14 \%$ of lymphocytes; $p=0.049$ ). In contrast to this observation, we detected a lower percentage of $\mathrm{CD} 4+\mathrm{T}$ cells in responders compared with nonresponders (27.8\% of lymphocytes vs. $57.9 \% ; p=0.07$ ). In addition, there was a higher percentage of $\mathrm{T}$ cells in patients than in $\mathrm{HC}$ (75.5\% of lymphocytes vs. $68.3 \% ; p=0.06$ ) but lower percentages of B cells in patients compared with $\mathrm{HC}$ (5.7\% of lymphocytes vs. $10.6 \% ; p=0.015)$. At screening, the proportion of T cells and monocytes expressing PD-1 (\%) as well as their matching ligands PD-L1 and PD-L2 on blasts and monocytes in $\mathrm{PB}$ was significantly higher in patients compared with $\mathrm{HC}(p<0.01$, Fig. 1c, d), suggesting an exhausted T-cell immune status in these HMA-failure patients prior to treatment initiation. During TAL therapy, PD-L1 expression on $\mathrm{T}$ cells further increased significantly (from 9.6 to $16.0 \%$; $p=0.047$ ), but we could not detect any significant differences between responders and nonresponders.

MDSCs are markedly expanded in the BM of MDS patients, playing a key pathogenetic role in the development of ineffective hematopoiesis and disease progression, while enhancing immunosuppression by inhibiting T- and NKcell activation [16]. Expression (\%) of CD123 on immature MDSC (iMDSC) was higher in $\mathrm{HC}$ than in patients (Fig. 1e) and pre-treatment expression (MFI) of CD123 on iMDSCs was higher in responders than in nonresponders (2565 vs. 302; $P=0.07$ ). MDSCs with high CD123 expression might have been more susceptible to NK-mediated killing after TAL, resulting in enhanced T- and NK-cell activation and subsequent increase in ADCC lysis of BM blasts. Corresponding to this observation, we observed in all patients a negative correlation between the percentage of NK cells and iMDSCs $(r-0.44 ; p=0.031)$. Anti-CD123 targeted therapy resulted in decreased CD123 + MFI (4240 vs. 2910; $p=0.004)$ on PB iMDSCs and furthermore in a reduction of CD123-positive iMDSC in BM (0.08 vs. $0.02 \%$ of leukocytes, $p=0.05$ ). At baseline, patients showed a higher expression of CD123 + BM blasts compared with HC (MFI 227 vs $170, p=0.06$ ) (Fig. 1f). However, there were no significant differences in CD123 expression on BM blasts between responders and nonresponders.

To conclude, our data indicate that blockade of CD123 was clinically less effective in this advanced study population including HR-MDS and AML patients resistant to previous HMA therapy. Patients already displayed significant alterations in their NK and T-cell repertoire prior to study treatment. Most importantly, there was significant toxicity that led to a high rate of early treatment 
A Blast count change in $\mathrm{BM}(\%)$

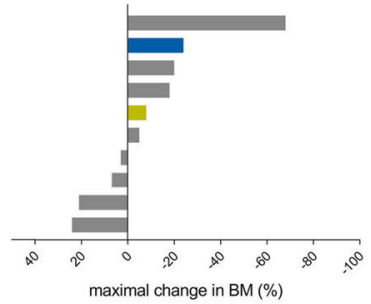

B

CD56dim/CD16+ of NK-cells

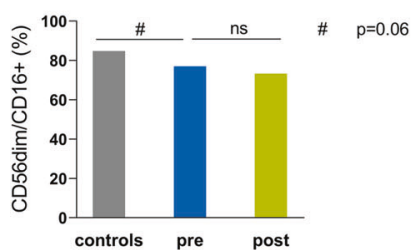

C

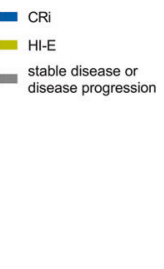

stable disease or
disease progressio

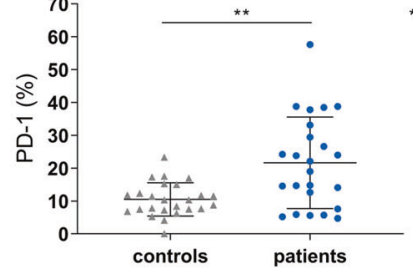

D



E

** $p=0.008$



$\mathbf{F}$

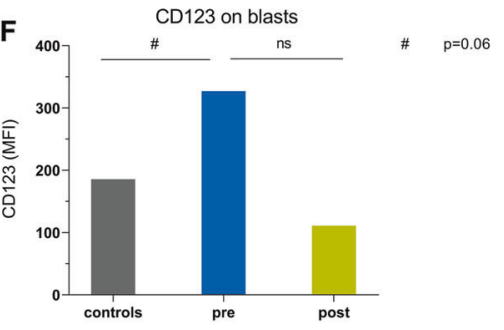

Fig. 1 a Blast count change in BM of patients during therapy. Maximal change of BM blast count (\%) from baseline of available patients who reached response assessment after 3 months of TAL treatment $(n=10)$. b Distribution of mature NK cells in patients compared with HC. At baseline, patients expressed lower levels of CD56dim NK cells compared with $\mathrm{HC}$ in PB. $(p=0.065)$ (pretreatment (pre); posttreatment (post)), patients $n=24, \mathrm{HC}=24$. $\mathbf{c}$ Expression of PD-1 on lymphocytes in patients compared to HC. During screening, expression of PD-1 on lymphocytes in $\mathrm{PB}$ was significantly higher in patients compared to $\mathrm{HC}$. patients $n=24, \mathrm{HC}=24$. d Expression of PD-L1 on blasts in patients compared with HC. Expression of the corresponding ligand PD-L1 on blasts was significantly higher in patients than in HC. patients $n=24$,
$\mathrm{HC}=24$. e Expression of CD123 on iMDSCs in patients compared with HC. Pre treatment expression (percentage) of CD123 on immature MDSC (iMDSC) was higher in HC than in patients. A reduction of CD123+ iMDSC $(p=0.07)$ was detected during treatment. We determined iMDSC as LIN-, HLA-DR-, CD11b+, and CD33+. Patients baseline $n=24$, $\mathrm{HC} n=24$, patients post treatment $n=10$. Analysis for patients pre and post treatment was undertaken with those patients who reached three months of therapy $(n=10)$. f Expression of CD123 on BM blasts in patients compared with HC. Patients showed higher expression (MFI) of CD123 on BM blasts compared with HC. Under treatment, those MFI levels showed a nonsignificant reduction. (pretreatment (pre); posttreatment (post)) patients $n=24, \mathrm{HC}=24$ discontinuation and disease progression. Our results demonstrate an unfavorable risk/benefit profile of single agent TAL, thus successful development of an anti-CD123 therapeutic approach for myeloid malignancies is likely to require the addition of cytotoxic payloads like antibody drug conjugates or the utilization of further synergistic immune modalities such as CD123-specific chimeric antigen receptor modified T- or NK cells in order to improve potency.

Acknowledgements The authors thank Michael Cross for manuscript editing and constructive discussions during the development of the manuscript. The authors thank all patients who participated in this study.

Funding The study was sponsored by Janssen Research \& Development, LLC.

Author contributions UP designed the study, UP wrote and conducted the clinical protocol. UP, UO, AK, and FS collected, analyzed, and interpreted the data; UP, AK, FS and UO wrote the paper. All other authors provided patient support, collected data, and edited the paper.

\section{Compliance with ethical standards}

Conflict of interest Uwe Platzbecker, Research funding: Janssen Pharmaceuticals. Consultancy and Honoraria: Novartis, Abbvie,
Celgene. AG, Research funding: Janssen Pharmaceuticals. KG: Research funding: Janssen Pharmaceuticals. Advisory committee: Abbvie. MP: Research funding: Janssen Pharmaceuticals. CW: Research funding: Janssen Pharmaceuticals. PF: Research funding: Janssen Pharmaceuticals. Richard Schlenk, Honoraria and Research Funding: Teva Pharmaceutical Industries. CT, Employment and Equity Ownership: AgenDix GmbH, Consultancy, Honoraria, Research Funding and Speakers Bureau: Novartis, Honararia: Daiichi Sankyo. LA: Research funding: Janssen Pharmaceuticals. Consultancy and Honoraria: Celgene, Novartis, Takeda Jazz, Abbvie. UO: Research funding: Janssen Pharmaceuticals. ASK, FS, JK, KS, JMM, FC, SG, DW, SS, and AM declare no relevant conflicts of interests.

Publisher's note Springer Nature remains neutral with regard to jurisdictional claims in published maps and institutional affiliations.

\section{References}

1. Nazha A, Sekeres MA. Precision medicine in myelodysplastic syndromes and leukemias: lessons from sequential mutations. Annu Rev Med. 2017;68:127-37.

2. Platzbecker U. Treatment of MDS. Blood 2019;133:1096-107.

3. Komrokji RS. Treatment of higher-risk myelodysplastic syndromes after failure of hypomethylating agents. Clin Lymphoma Myeloma Leuk. 2015;15:S56-9.

4. Testa U, Pelosi E, Frankel A. CD 123 is a membrane biomarker and a therapeutic target in hematologic malignancies. Biomark Res. 2014;2:4. 
5. Jin L, Lee EM, Ramshaw HS, Busfield SJ, Peoppl AG, Wilkinson L, et al. Monoclonal antibody-mediated targeting of CD123, IL-3 receptor alpha chain, eliminates human acute myeloid leukemic stem cells. Cell Stem Cell. 2009;5:31-42.

6. Graf M, Hecht K, Reif S, Pelka-Fleischer R, Pfister K, Schmetzer $\mathrm{H}$. Expression and prognostic value of hemopoietic cytokine receptors in acute myeloid leukemia (AML): implications for future therapeutical strategies. Eur J Haematol. 2004;72:89-106.

7. Xie LH, Biondo M, Busfield SJ, Arruda A, Yang X, Vairo G, et al. CD123 target validation and preclinical evaluation of ADCC activity of anti-CD123 antibody CSL362 in combination with NKs from AML patients in remission. Blood Cancer J. 2017;7:e567.

8. Jordan CT, Upchurch D, Szilvassy SJ, Guzman ML, Howard DS, Pettigrew AL, et al. The interleukin-3 receptor alpha chain is a unique marker for human acute myelogenous leukemia stem cells. Leukemia. 2000;14:1777-84.

9. Schurch CM. Therapeutic antibodies for myeloid neoplasmscurrent developments and future directions. Front Oncol. 2018;8:152.

10. Busfield SJ, Biondo M, Wong M, Ramshaw HS, Lee EM, Ghosh $\mathrm{S}$, et al. Targeting of acute myeloid leukemia in vitro and in vivo with an anti-CD123 mAb engineered for optimal ADCC. Leukemia. 2014;28:2213-21.
11. Cuesta-Mateos C, Alcaraz-Serna A, Somovilla-Crespo B, MunozCalleja C. Monoclonal antibody therapies for hematological malignancies: not just lineage-specific targets. Front Immunol. 2017;8:1936.

12. Cheson BD, Greenberg PL, Bennett JM, Lowenberg B, Wijermans PW, Nimer SD, et al. Clinical application and proposal for modification of the International Working Group (IWG) response criteria in myelodysplasia. Blood. 2006;108:419-25.

13. Montesinos P, Roboz G, Bulabois CE, Subklewe M, Platzbecker U, Ofran Y, et al. Safety and efficacy of talacotuzumab plus decitabine or decitabine alone in patients with acute myeloid leukemia not eligible for chemotherapy: results from a multicenter, randomized, phase $2 / 3$ study. Leukemia. 2019. In press.

14. Lanier LL. NK cell recognition. Annu Rev Immunol. 2005;23: 225-74.

15. Epling-Burnette PK, Bai F, Painter JS, Rollison DE, Salih HR, Krusch M, et al. Reduced natural killer (NK) function associated with high-risk myelodysplastic syndrome (MDS) and reduced expression of activating NK receptors. Blood. 2007;109:4816-24.

16. Bronte V, Brandau S, Chen SH, Colombo MP, Frey AB, Greten $\mathrm{TF}$, et al. Recommendations for myeloid-derived suppressor cell nomenclature and characterization standards. Nat Commun. 2016; 7:12150. 